Journal Club

Editor's Note: These short, critical reviews of recent papers in the Journal, written exclusively by graduate students or postdoctoral fellows, are intended to summarize the important findings of the paper and provide additional insight and commentary. For more information on the format and purpose of the Journal Club, please see http://www.jneurosci.org/misc/ifa_features.shtml.

\title{
Stimulating the Semantic Network: What Can TMS Tell Us about the Roles of the Posterior Middle Temporal Gyrus and Angular Gyrus?
}

\author{
Sarah Tune and Salomi S. Asaridou \\ Department of Neurology, University of California, Irvine, California 92697-4545 \\ Review of Davey et al.
}

Semantic knowledge plays a crucial role in guiding our everyday actions and lies at the heart of human communication. The availability of modern neuroimaging techniques has resulted in a vast number of studies that have advanced our understanding of different aspects of semantic processing. Whereas there is a general consensus that semantic cognition is supported by a distributed neural network, the specific contributions of various brain regions and circuits remain controversial (Hoffman et al., 2015). Among the brain regions thought to engage in semantic processing, various parts of the left anterior temporal lobe (ATL), posterior middle temporal gyrus (pMTG), and angular gyrus (AG) have emerged as candidates for higherorder convergence zones or network hubs (Patterson et al., 2007; Binder and Desai, 2011). Attempts to characterize the role(s) of these regions have sparked lively debates and led to different models of the semantic network.

Although there is general agreement that the ATL is engaged in the representation of taxonomic associations (relationships based on features and hierarchically

Received Jan. 18, 2016; revised March 4, 2016; accepted March 4, 2016. The authors declare no competing financial interests.

Correspondence should be addressed to Dr. Sarah Tune, Department of

Neurology University of California, Irvine Biological Sciences III, Irvine, CA 92697-4545.E-mail:tunes@uci.edu.

DOI:10.1523/JNEUROSCI.0194-16.2016

Copyright $\odot 2016$ the authors $\quad 0270-6474 / 16 / 364405-03 \$ 15.00 / 0$ organized: eg, bulldog, boxer, and corgi are members of the superordinate category "dogs"), the role of temporoparietal areas, such as the AG and pMTG, is still under debate. According to one view, the AG (and potentially the pMTG) is complementing the ATL by capturing thematic associations between concepts (relationships based on a given theme: eg, wolf, sheep, and shepherd are part of a sheep farm; Schwartz et al., 2011; de Zubicaray et al., 2013). This view is motivated by lesion studies which demonstrate that patients with ATL lesions make more taxonomic errors, whereas patients with temporoparietal lesions make more thematic errors (Schwartz et al., 2011). An alternative view argues for distinct roles of AG and pMTG in automatic and controlled semantic retrieval processes, respectively (Whitney et al., 2011a,b, 2012; Humphreys and Lambon Ralph, 2015). This alternative view is motivated by the finding that the two regions show distinct functional connectivity profiles at rest and respond differently during difficult semantic tasks. The left AG is considered part of the default mode network, and shows a stronger decrease in neural activity between rest and difficult semantic tasks than between rest and easy tasks. In contrast, the pMTG is functionally connected to regions of the frontoparietal control system and shows increased neural activity during semantically demanding tasks.
Davey et al. (2015) applied inhibitory repetitive transcranial stimulation (rTMS) to the left ATL, pMTG and mid-AG to elucidate the role of these regions in: (1) representation versus retrieval of semantic knowledge, (2) processing of taxonomic versus thematic associations, and (3) controlled versus automatic retrieval of information. One of the key findings was a dissociation of mid-AG and pMTG that suggests these regions make distinct contributions to automatic and controlled semantic retrieval. No significant TMS-induced changes in behavior were found for the ATL.

Davey et al. (2015) examined how rTMS affected performance on two tasks in which participants were asked to match a picture to one of three printed words. In the thematic task, participants were asked to select the word that was thematically associated with the probe picture. The correct picture-word matches were either strongly associated (eg, a picture of a trombone and the word "orchestra") or weakly associated (eg, a picture of a pair of slippers and the word "hospital"). In the taxonomic task, participants selected the word that described the picture at either a specific or a superordinate level. For the specific level, the three words were competing members of the same semantic category (eg, bulldog, boxer, corgi), whereas for the superordinate level the words were from different semantic categories (eg, animal, tool, plant). 
In line with their hypothesis that pMTG and mid-AG are involved in different types of retrieval, the authors predicted that rTMS of pMTG, but not AG, would impair performance on the thematic task, and this impairment would be more pronounced for weak than for strong thematic associations given that the former requires more semantic control than the latter. In addition, they predicted that stimulation of pMTG, but not mid-AG, would affect processing of both superordinate and specific taxonomic associations, arguing that both require some degree of semantic control. Moreover, they reasoned that if mid-AG and/or pMTG are involved solely in the representation of thematic knowledge, then rTMS to these regions should not affect performance on the taxonomic task.

Overall, rTMS led to longer reaction times, whereas response accuracy was not significantly affected. The authors examined the disruptive effect of rTMS in three separate analyses using linear mixed effect models. A first global analysis investigated the effect of rTMS across all tasks and stimulation sites. Pairwise comparisons of least square means derived from the model indicate that, contrary to the authors' predictions, rTMS to mid-AG led to significantly longer reaction times on both levels of the thematic task and on the specific level of the taxonomic task. rTMS to pMTG increased reaction times for superordinate and specific taxonomic matching, only. Two follow-up analyses examined the effects separately for each matching task. For the thematic task, a more fine-grained measure of association strength, as well as additional covariates accounting for the lexical frequency, polysemy, and familiarity of the correct target word were included in the model. The results of this analysis confirmed the authors' hypothesized dissociation: whereas rTMS to pMTG disrupted the matching of weakly associated pairs, stimulation of mid-AG disrupted strongly associated pairs. Finally, the follow-up analysis on the taxonomic task included covariates accounting for concept typicality and familiarity of the picture stimulus and found results comparable to those of the first analysis. The authors concluded that the observed patterns provide strong evidence for the hypothesis that pMTG contributes to controlled retrieval, whereas mid-AG is involved in automatic retrieval of semantic information.

The observed dissociation of mid-AG and pMTG based on the disruptive effect of rTMS on the thematic-matching task is a potentially important result, but the striking differences in the results of the global analysis and the follow-up thematic-task analysis are difficult to reconcile. Both analyses appear to indicate a dissociation of mid-AG and pMTG for a task that involved identifying the thematic link between two concepts; however, they revealed different dissociations: according to the global analysis, rTMS on mid-AG but not pMTG affected performance in the thematic task, whereas according to the follow-up analysis rTMS on pMTG did affect performance, specifically for the most difficult, weak thematic associations. Given the level of description, it is difficult to discern what is driving these differences. The analyses differ in how association strength scores were obtained and modeled, as well as in the set of included covariates and random effects. A more thorough report of the model fitting, comparison procedure, and statistical results might have made the findings easier to interpret.

Although TMS studies allow controlled stimulation of multiple brain sites within the same healthy subject and enable researchers to discern the functions of distinct regions, TMS, and especially rTMS, does come with certain limitations. First, observed dissociations provide only limited insight into the underlying mental processes and their neural implementation. Conclusions about the neural underpinnings of specific tasks critically hinge on the set of mental processes one assumes necessary for task execution, and on how such processes can be linked to the brain mechanisms and circuits involved in the task. We feel that in the current study, the mapping between the tasks and the underlying processes they are supposed to tap was not always clear enough. For example, successful retrieval of a picture-word association is impossible without the activation of the respective semantic representations. It is therefore unclear how changes in behavior following rTMS could have differentiated between representation and retrieval accounts. The use of an additional passive priming task, not requiring explicit semantic judgments and consequently no controlled retrieval, could have helped to tease apart the competing accounts. Moreover, any conclusions about the involvement of mid-AG and pMTG in semantic control are obscured by the fact that the tasks did not only call for varying degrees of semantic control but might have relied on qualitatively different control processes. For example, for the specific level of the taxonomic task, participants had to select among highly similar members of the same semantic category. A correct match therefore required the suppression of competing alternatives. The identification of semantic associations in the thematic task, especially for weakly associated pairs, did not involve a selection among competing concepts and may have required a different kind of semantic control. Matching the tasks on both the degree and the type of semantic control would have allowed for stronger conclusions.

A second caveat regarding interpretation of rTMS studies is that low-frequency off-line rTMS leads to a reduction in excitability in not only the targeted cortical area but also in interconnected cortical and even subcortical areas (Siebner and Rothwell, 2003; Blankenburg et al., 2008). For the current study, this limits the conclusions that can be drawn about the dissociable contribution of single, discrete brain regions, such as the mid-AG or pMTG. Both areas are located in complex zones of association cortex characterized by its various connections to several major white matter pathways and a confluence of distinct neural systems. Sections of the middle temporal lobe directly anterior to the pMTG share functional connections with the mid-AG, and parts of the inferior parietal lobule adjacent to the mid-AG are included in the same inferior parietal network as the pMTG (Yeo et al., 2011). It is therefore questionable whether rTMS can cause a virtual lesion to one without influencing the other, either directly or indirectly (in terms of information flow).

A final caveat relates to the impact of inhibitory rTMS on regions with different activation profiles. Areas of the default network, such as the mid-AG, tend to show a decrease in neural activity for active tasks compared with rest, whereas the pMTG exhibits the opposite pattern. In light of these known physiological differences, one may question the assumption that TMS to these regions would lead to qualitatively comparable disruptive effects. In fact, judging from the follow-up analysis of the thematic task, it appears that at least in some of the weakly correlated pairs, rTMS to the AG resulted in shorter reaction times compared with the no-TMS baseline session. In the absence of supplemental neuroimaging data, the nature of the impact of rTMS on the two areas under investigation remains speculative.

The fact that the effects of rTMS are not restricted to the primary site of stimulation is a limitation that is difficult to overcome. This feature however makes it an excellent tool for investigating changes in functional connectivity within a 
distributed neural network. Its apparent weakness can be turned into a strength by combining rTMS with fMRI and by focusing on the link between behavioral and functional-connectivity changes (Allen et al., 2007; Siebner et al., 2009; Watanabe et al., 2015). This would shift the emphasis from the role of discrete areas in the encoding or retrieval of semantic knowledge to questions about the mechanistic principles and the neural implementation of semantic cognition.

\section{References}

Allen EA, Pasley BN, Duong T, Freeman RD (2007) Transcranial magnetic stimulation elicits coupled neural and hemodynamic consequences. Science 317:1918-1921. CrossRef Medline

Binder JR, Desai RH (2011) The neurobiology of semantic memory. Trends Cogn Sci 15: 527-536. CrossRef Medline

Blankenburg F, Ruff CC, Bestmann S, Bjoertomt O, Eshel N, Josephs O, Weiskopf N, Driver J (2008) Interhemispheric effect of parietal TMS on somatosensory response confirmed directly with concurrent TMS-fMRI. J Neurosci 28:13202-13208. CrossRef Medline

Davey J, Cornelissen PL, Thompson HE, Sonkusare S, Hallam G, Smallwood J, Jefferies E (2015) Automatic and controlled semantic retrieval: TMS reveals distinct contributions of posterior middle temporal gyrus and angular gyrus. J Neurosci 35:15230-15239. CrossRef Medline de Zubicaray GI, Hansen S, McMahon KL (2013) Differential processing of thematic and categorical conceptual relations in spoken word production. J Exp Psychol Gen 142:131-142. CrossRef Medline

Hoffman P, Jefferies B, Ralph ML (2015) Special issue of neuropsychologia: semantic cognition. Neuropsychologia 76:1-3. CrossRef Medline

Humphreys GF, Lambon Ralph MA (2015) Fusion and fission of cognitive functions in the human parietal cortex. Cereb Cortex 25: 3547-3560. CrossRef Medline

Patterson K, Nestor PJ, Rogers TT (2007) Where do you know what you know? The representation of semantic knowledge in the human brain. Nat Rev Neurosci 8:976-987. CrossRef Medline

Schwartz MF, Kimberg DY, Walker GM, Brecher A, Faseyitan OK, Dell GS, Mirman D, Coslett HB (2011) Neuroanatomical dissociation for taxonomic and thematic knowledge in the human brain. Proc Natl Acad Sci U S A 108:8520-8524. CrossRef Medline

Siebner HR, Rothwell J (2003) Transcranial magnetic stimulation: new insights into representational cortical plasticity. Exp Brain Res 148:1-16. CrossRef Medline

Siebner HR, Bergmann TO, Bestmann S, Massimini $M$, Johansen-Berg $H$, Mochizuki $H$, Bohning DE, Boorman ED, Groppa S, Miniussi C, Pascual-Leone A, Huber R, Taylor PC, Ilmoniemi RJ, De Gennaro L, Strafella AP, Kähkönen S, Klöppel S, Frisoni GB, George MS, et al. (2009) Consensus paper: combining transcranial stimulation with neuroimaging. Brain Stimul 2:58-80. CrossRef Medline
Watanabe T, Hanajima R, Shirota Y, Tsutsumi R, Shimizu T, Hayashi T, Terao Y, Ugawa Y, Katsura M, Kunimatsu A, Ohtomo K, Hirose S, Miyashita Y, Konishi S (2015) Effects of rTMS of pre-supplementary motor area on fronto basal ganglia network activity during stop-signal task. J Neurosci 35:4813-4823. CrossRef Medline

Whitney C, Jefferies E, Kircher T (2011a) Heterogeneity of the left temporal lobe in semantic representation and control: priming multiple versus single meanings of ambiguous words. Cereb Cortex 21:831-844. CrossRef Medline

Whitney C, Kirk M, O’Sullivan J, Lambon Ralph MA, Jefferies E (2011b) The neural organization of semantic control: TMS evidence for a distributed network in left inferior frontal and posterior middle temporal gyrus. Cereb Cortex 21:1066-1075. CrossRef Medline

Whitney C, Kirk M, O’Sullivan J, Lambon Ralph MA, Jefferies E (2012) Executive semantic processing is underpinned by a large-scale neural network: revealing the contribution of left prefrontal, posterior temporal, and parietal cortex to controlled retrieval and selection using TMS. J Cogn Neurosci 24:133-147. CrossRef Medline

Yeo BT, Krienen FM, Sepulcre J, Sabuncu MR, Lashkari D, Hollinshead M, Roffman JL, Smoller JW, Zöllei L, Polimeni JR, Fischl B, Liu H, Buckner RL (2011) The organization of the human cerebral cortex estimated by intrinsic functional connectivity. J Neurophysiol 106:1125-1165. CrossRef Medline 